\title{
Plan estratégico de innovación en el área de producción para el mejoramiento de la rentabilidad económica de la Industria Avícola
}

\section{Strategic plan for innovation in the production area to improve the economic profitability of the Poultry Industry}

\author{
Enma Reyes Romo ${ }^{1}$ \\ sussyreyes 1981@hotmail.com \\ 0000-0003-2166-6437 \\ Carlos Carrera Montalvo 2 \\ ccarrera@uteg.edu.ec \\ 0000-0003-0580-4027
}

Fecha de recibo: 1/12/2020; Fecha de aceptación: 4/3/2020

\begin{abstract}
RESUMEN
En el proceso de innovación es común introducir novedades en las empresas, dichas novedades pueden modificar elementos que ya existen o introducir elementos totalmente nuevos a la empresa, es decir, se puede renovar considerando recursos que existen, logrando mejorarlos o implementando nuevos. Con la innovación se busca producir más productos para obtener más utilidad, logrando posicionamiento y competitividad en el mercado y también optimizar recursos como mano de obra e insumos, para ello, existen muchos tipos de innovación como la innovación empresarial, la disruptiva, la educativa, creatividad y, la tecnológica. El presente artículo expone el diseño de un plan estratégico de innovación para mejorar la rentabilidad económica de la industria avícola, que se desarrolló en el cantón Montecristi, provincia de Manabí Ecuador; evidenciando lo principales problemas en el área de producción. El diseño de la investigación es cuantitativo con un nivel correlacional - no experimental, utilizando la entrevista como instrumento de recopilación de datos, que fue realizada a nivel directivo empresarial y de diferentes áreas de producción como granja reproductora, alimento balanceado e incubación de la industria avícola. Se logró proponer un plan estratégico de innovación productiva, además se encontró fortalezas por la experiencia del personal operativo.
\end{abstract}

Palabras clave: Plan estratégico, innovación, competitividad, rentabilidad, avicultura

\footnotetext{
${ }^{1}$ MAE, Universidad Tecnológica Empresarial de Guayaquil, Ecuador

${ }^{2}$ Magister en Administración de Empresas, Universidad Tecnológica Empresarial de Guayaquil, Ecuador
} 


\begin{abstract}
In the innovation process, it is common to introduce new features in companies, these developments can modify elements that already exist or introduce totally new elements to the company, that is, it can be renewed considering existing resources, improving them or implementing new ones. Innovation seeks to produce more products to obtain more utility, achieving positioning and competitiveness in the market and also optimizing resources such as labor and supplies, for this, there are many types of innovation such as business innovation, disruptive, educational, creativity and technology. This article presents the design of a strategic innovation plan to improve the economic profitability of the poultry industry, which was developed in the Montecristi canton, Manabí province - Ecuador; evidencing the main problems in the production area. The research design is quantitative with a correlational - nonexperimental level, using the interview as a data collection instrument, which was carried out at the business management level and from different production areas such as breeding farm, balanced feed and incubation of the poultry industry. It was possible to propose a strategic plan for productive innovation and strengths were also found due to the experience of the operating personnel.
\end{abstract}

Keywords: Strategic plan, innovation, competitiveness, profitability, poultry

\title{
Introducción
}

La planeación estratégica es un proceso adoptado por la empresa basadas en su organización administrativa, con el fin de evaluar la situación de la institucional y proponer estrategias que mejoren su viabilidad (Quezada, Neri, \& Pérez, 2015). Según Rio (2018) en la planificación estrategia se consideran los objetivos estratégicos relacionados directamente con la misión, una evaluación situacional de la empresa, a donde se debe ir y en base a la evidencia empírica cuales la situación probable a lograrse y de esta manera determinar el camino que se puede lograr. La planeación estratégica incluye seis componentes que son estructurales; quien son los que van a cumplir con ejecución de las estrategias, el direccionamiento que se va a cumplir, el diagnóstico de la organización, el análisis de escenarios y resultados posibles, la formulación y determinación de la mejora estrategia y la auditoria estratégica que en otras palabras es el control. Al mencionarse la planificación estratégica se tiene que esta influye en la toma de decisiones de la empresa para de esta manera lograr el mejor proceder de la organización fundamentadas en las medidas que se están desarrollando (Robayo, 2016).

Quezada, Neri, \& Pérez (2015) menciona los elementos de la planificación estratégica, siendo la visión, a lo que quiere llegar la empresa en un plazo determinado, la misión que se fundamenta en lo que realiza la empresa. El análisis externo de la empresa proporciona información de los competidores que se encuentran en el mercado y esto permite fijar precios relacionados o incluso de esta manera conocer el accionar de los competidores e implementar estrategias competitivas en la organización. 


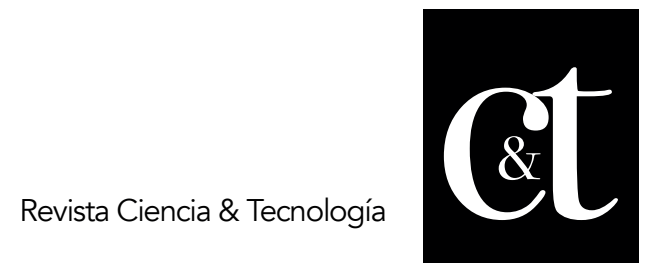

No. 26, 30 de abril de 2020

ISSN impreso: 1390 - 6321

ISSN online: 2661 - 6734

La visión permite enfocarse en lo que se quiere llegar como organización esto permite direccionar a la empresa de una manera más eficiente, es el propósito a mediano o largo plazo de la empresa que mediante el cumplimiento de actividades gestionadas bajo la planificación se puede lograr en un tiempo determinado mejoras situacionales que aumenten el posicionamiento o situación de la empresa. La misión permite responder a las preguntas relacionadas a lo que realiza el negocio, cual es el negocio, quienes son los clientes, cuáles son los objetivos de la empresa, es fundamental que el personal conozca la empresa para así visualizar y cumplir sus funciones adecuadamente. Países como Brasil, presentan uno de los menores costos de producción en base a una buena infraestructura empresarial que ha generado una ventaja competitiva a nivel mundial, contando con materia prima e insumos a precios bajos (Poultry, 2019).

\section{Metodología y herramientas de análisis estratégico}

Aponte (2015) afirma que los científicos hacen mucho énfasis en la investigación y la relacionan con el desarrollo de la organización para redescubrir nuevas formas de producción y de productos que permitan la satisfacción de bienes y servicios de los individuos. El diseño del estudio es considerado con un enfoque mixto cualicuantitativo para realizar un análisis situacional a nivel organizativo y estructural de la empresa GENETSA asentada en el cantón Montecristi de la provincia de Manabí. Para determinar la situación actual de la compañía se utilizó una metodología de carácter descriptivo y explicativo. La condición descriptiva se da en el desarrollo de la investigación documental de los datos que existen en la compañía y a través del método inductivo - deductivo, con el fin de obtener una valoración sustancial de la condición en la que se encuentra la misma. Como instrumentos de investigación se desarrolló una entrevista al equipo directivo de la compañía GENETSA, para lo que se solicitó la autorización debida.

En lo que respecta al método analítico en la investigación se logró determinar, analizar y ordenar minuciosamente los datos que se obtuvieron en los cantones de la zona norte de Manabí, uno de ellos fueron las ventas que han transcurrido dentro del período de investigación, información que sirvió para comparar el análisis interno y externo a través de las preguntas de la entrevista y encuesta, donde se mencionan las fortalezas, oportunidades, debilidades y amenazas de cada una de las empresas.

La población de GENETSA es de 204 trabajadores que laboran en los diferentes procesos productivos de la cría de pollos, para lo cual se seleccionó una muestra representativa de la población mediante la fórmula del tamaño de la muestra, estableciéndose en un total de 133 trabajadores. Se aplicó las encuestas al personal de GENETSA para conocer su situación en base a la aplicación de un plan estratégico y los procesos que se ejecutan en esta empresa. Se procesó los datos a través del software estadístico SPSS, para luego realizar histogramas de frecuencias de los resultados obtenidos, hallando información relevante sobre el conocimiento del personal y la viabilidad de la aplicación de un plan estratégico en la empresa. 


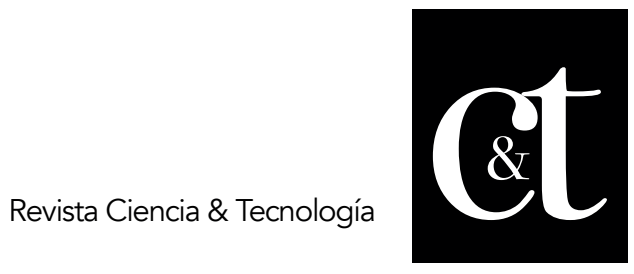

No. 26, 30 de abril de 2020

ISSN impreso: 1390 - 6321

ISSN online: 2661 - 6734

\section{Indicadores de Gestión}

El desarrollo de nuevos productos incursiona en las necesidades actuales del mercado y en la innovación de recursos materiales y equipos que facilitan la satisfacción de necesidades de las personas (Aillón, 2012). El establecimiento de las estrategias se delude en la creación de planes de acción que permiten lograr un objetivo concreto mediante medidas, actividades y acciones en la organización. En la planificación estratégica los indicadores de gestión son elementos que permiten realizar el seguimiento y el control de la empresa, esta medición de cumplimiento debe realizarse de manera continua para poder verificar el avance y control de las actividades en la organización (Hinojosa, 2006). Los indicadores de gestión son externos como es el caso de nivel de satisfacción al cliente e internos, siendo un ejemplo claro el nivel de ingresos de los empleados. Los indicadores pueden medir la eficacia y eficiencia en la organización, considerando por ejemplo el número de artículos entregados, cantidad de clientes y tiempo de espera, así como el nivel de cumplimiento de los procesos.

\section{La industria avícola en el Ecuador}

La carne de pollo es parte de la pirámide de alimentos que las personas deben consumir normalmente contiene nutrientes y proteínas necesarias en mejor proporción que otros tipos de carne. En el Ecuador, el pollo es parte de la canasta básica puesto que sus precios son accesibles y se pueden conservar fácilmente. Los inicios de la avicultura son en 1957 cuando se instauró la primera planta de incubación artificial en la Ciudad de Quito, sin embargo, se da mayor hincapié en 1970, puesto que se empiezan a expandir a las principales provincias del Ecuador. Esto se da a partir del boom petrolero que, según la revista Productor Agropecuario (2019), inyecta dinero en la economía lo que fomenta la inversión.

El Ecuador paso de una producción de 50 millones de aves en 1990 a 233,5 millones en el año 2014, esto evidencia que el nivel de crecimiento es del $400 \%$ en este periodo generando un nivel de empleo y seguridad económica y alimentaria mediante la producción de pollo a nivel nacional (Santillán, 2014). A nivel nacional existe producción avícola en todas las provincias del Ecuador, se tiene que al año se producen 220 pollos y se logró un crecimiento muy elevado en relación a la década anterior. En el Ecuador se tiene que la CONAVE agrupa al $75 \%$ de productores de carne de pollo y que se considera que los ecuatorianos consumen $32 \mathrm{~kg}$ de pollo al año, luego consume carne de res y finalmente la carne de cerdo.

Ecuador tiene 4 regiones con climas diferentes, sin embargo, la región más viable y mejor aprovechada para la cría de pollo es la costa por sus temperaturas y condiciones climáticas, que permiten que la crianza del pollo sea rápida teniendo un aproximado de 42 días en total como ciclo productivo (Galeano, 2014). En el caso de la producción de huevos es más representativo en ciertas partes de la sierra como es Quito y Cotopaxi, así también como en Manabí y en el resto de las zonas se han optado por diferentes medidas adaptadas a las condiciones de la región pero que con mucho cuidado permiten la crianza de pollos para consumo 
humano, teniendo que este sector es uno de los que mayor nivel de empleo ha generado.

El comportamiento del mercado de la carne de pollo y del huevo para consumo, se basa en la ley de la oferta y la demanda, lo que hace de las empresas avícolas negocios muy inestables y susceptibles a procesos de especulación, en donde los más fuertes son los que tienen el dominio total del mercado. Por otra parte, el consumidor ecuatoriano (ama de casa), prefiere un pollo pigmentado y grande, de aproximadamente 2,7 a $3 \mathrm{Kg}$. de peso en canal; así también demanda de un huevo con cascarón de color marrón, dejando al huevo blanco muy pocas oportunidades en el mercado (Barzallo, 2019). Se tiene que dada la importancia del sector avícola se han generado la necesidad de la instauración de controles por parte del gobierno a fin de evitar competencia desleal y asegurar el desarrollo adecuado de la industria avícola en el Ecuador (Calle, Estrada, \& Agudelo, 2015).

\section{El proceso de incubación artificial}

La utilización de innovación y tecnología en la crianza y producción de pollos radica en que en las últimas décadas se tecnificó mediante la creación de incubadoras artificiales, lo que ha permitido su expansión de una manera acelerada, puesto que en promedio cada gallina puede generar hasta 150 crías, y esto a su vez, permite tener $300 \mathrm{~kg}$ de carne disponible para el consumo (LLaque, 2009). La capacidad de producción en relación a volúmenes permite que en los últimos años la carne de pollo sea uno de los alimentos más relevantes de la canasta básica del Ecuador.

Los huevos producidos en granja pasan a la planta que permite la incubación de las crías en donde se seleccionan los huevos en base a su estado eliminando los que evidencian algún tipo de desperfecto. Los huevos listos para ser incubados son inicialmente almacenados en un cuarto frio con una temperatura de 18 grados pasando aproximadamente entre 1 a 6 días en este lugar (Jaramillo, 2017).

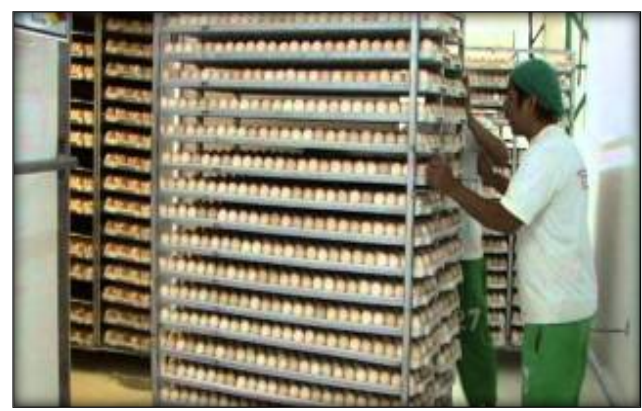

Gráfico 1. Ingreso de huevos al cuarto frio

Fuente: Genética Nacional S.A.

98

Revista científica Ciencia y Tecnología Vol 20 No 26 págs. 96-105 http://cienciaytecnologia.uteg.edu.ec 


\section{Incubación}

Para pasar a la fase de incubación los huevos son retirados del cuarto frio unas 12 horas antes para que no se produzca la mortalidad del embrión, cuando son colocados en las incubadoras por 18 días aproximadamente se mantienen a una temperatura de 36 grados, con humedad relativa y con cuidado y movimiento para que el embrión se encuentre en buenas condiciones en el interior del huevo.

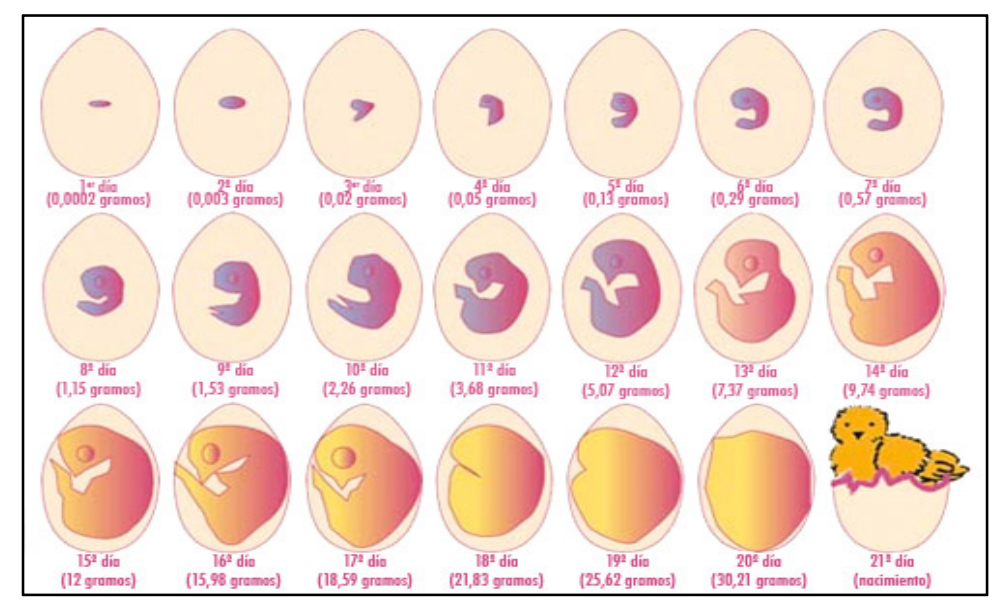

Gráfico 2. Ciclo evolutivo de un pollito bebe en 21 días

Fuente: Genética Nacional S.A.

\section{Transferencia}

Se procede luego de los 18 días a trasladar a los huevos con cuidado a gavetas que permiten que cuando nazcan no se caigan ni se lastimen, ubicándose de esta manera en máquinas nacederas. Los huevos pasan en este lugar por 3 días, con temperatura de 35 grados para luego empezar a eclosionar saliendo de su cascarón, tomando en cuenta que están naciendo el personal que controla este proceso lo retira para evitar deshidratación o muerte prematura (Robayo, 2016).

\section{Nacimiento}

Se tiene que luego de 18 días y 3 días más en las máquinas nacederas se logra que el $80 \%$ aproximadamente nazca sin ningún problema, sin embargo, el $20 \%$ por lo general no eclosiona, en donde el factor tiempo en la industria avícola es representativo, puesto que luego deben ser clasificados y contados para ser enviados a las granjas para ser criados y que logren un desarrollo máximo (Barbosa \& Dominique, 2012). 


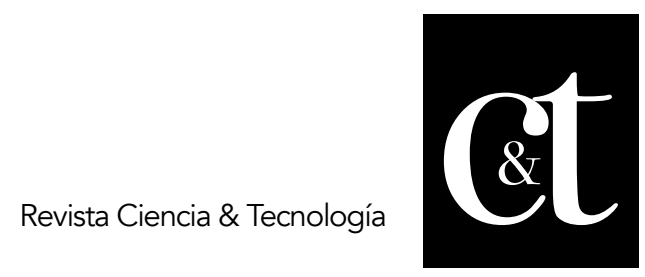

No. 26, 30 de abril de 2020

ISSN impreso: 1390 - 6321

ISSN online: 2661 - 6734

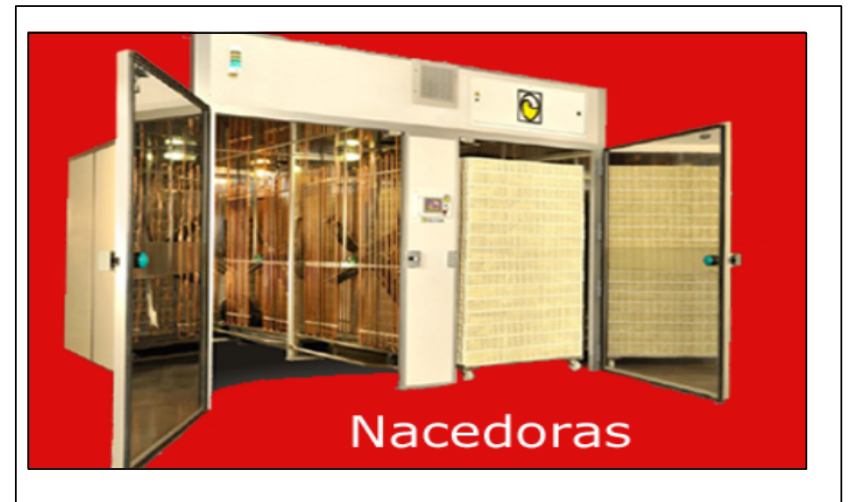

Gráfico 3. Máquina Nacedera

Fuente: Chick Master Company

\section{Transporte}

Finalmente, cuando ya se tienen a los pollos recién nacidos en cajas se los traslada en camiones acondicionados para la movilización de este tipo de producto, en donde el flujo de aire es un elemento fundamental del negocio, al llenar los camiones estos son dirigidos automáticamente a las granjas. Los camiones de traslado son climatizados.

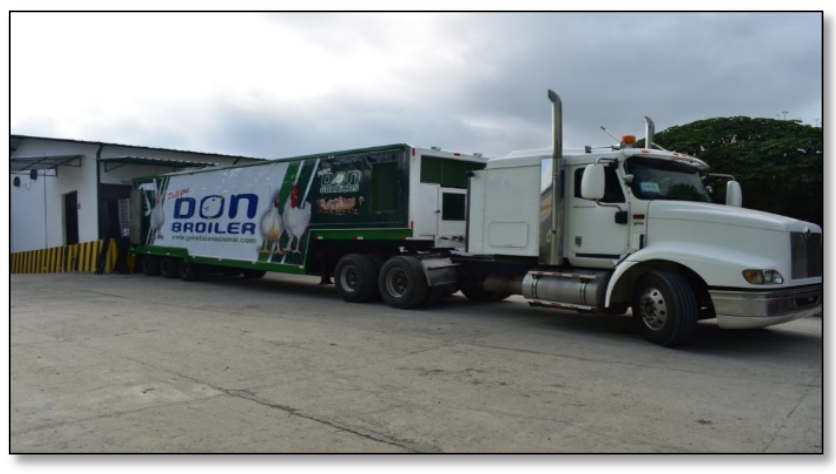

Gráfico 4. Máquina Nacedera

Fuente: Don Broiler

\section{Resultados y discusión}

La carne de pollo es una de las de mayor consumo a nivel mundial después de la carne de vacuno, hasta ahora es parte de la canasta familiar en muchos países por sus precios accesibles y su contenido vitamínicos. Según se prevé que la producción avícola crecerá alrededor del tres por ciento, además del consumo de 
huevos. Esto se puede dar a pesar de los altos costos de maquinarias y los procesos de crianza que requieren de un tiempo hasta obtener el producto final que son las aves de corral para el consumo.

GENETSA es una empresa encargada de la explotación de criaderos de pollos y reproducción de aves de corral, pollos y gallinas, aves de la especie Gallus Domesticus. En los últimos 4 años su nivel de utilidad ha sido positiva con un valor de $\$ 849.406,61$ en el 2016 para que en el año 2019 se llegó a una utilidad de $\$ 1.196 .567,23$ teniendo ya en el mercado aproximadamente 12 años en lo que respecta al sector avícola ecuatoriano.

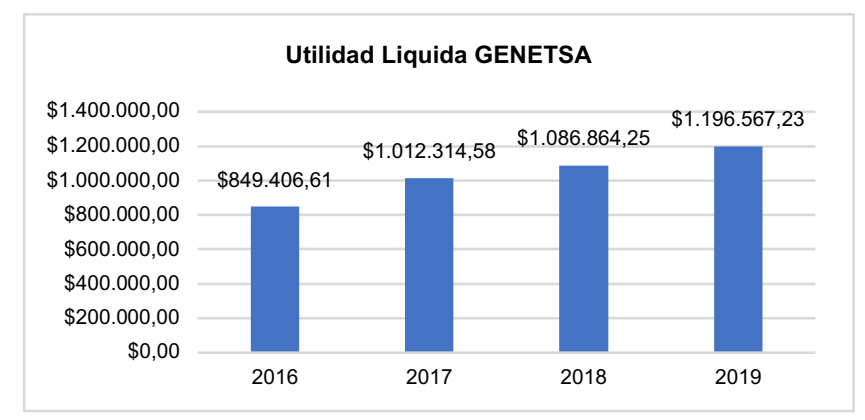

Gráfico 5. Utilidad GENETSA

Fuente: Empresa Avícola GENETSA

La población avícola en el año 1990 fue de 50 millones de aves, en cambio en el 2013 se obtuvo un total de 233 millones de aves. En el año 2019 se estima que un total de 250 millones de pollos de engorde aproximadamente. Esta es la proteína de mayor consumo de los ecuatorianos, según datos de la Asociación de Médicos Veterinarios Especialistas en Avicultura (Amevea) en Ecuador. Se estima que el consumo per cápita es de entre 30 y 32 kilogramos al año. La industria de producción de proteína animal que más ha crecido en estas dos décadas es la avícola. El ingreso bruto de la producción avícola en el país es de aproximadamente \$ 1.272 millones al año. Lo que equivale a una participación del $18 \%$ en el Producto Interno Bruto nacional. La comercialización de pollo involucra la producción de maíz amarillo duro, la materia prima para elaborar el balanceado para el sector avícola. Se calcula que la producción avícola consume 1,2 millones de toneladas métricas de maíz duro, esto equivale al $62 \%$ de lo que se produce en Ecuador. El consumo de pollo de los ecuatorianos hace 20 años era de 10 kilos por persona, cifra que se ha triplicado, afirmó Pérez. "Esto es beneficioso para el ser humano y, por otro lado, ha generado una industria importante en el sector de los alimentos, que está en crecimiento y se estima que puede ir más allá de lo que se ha alcanzado". La distribución de la industria es equitativa, de ahí que esté "casi en un $30 \%$ para cada sector, entre grandes, medianos y pequeños productores. Se calcula que en Ecuador hay un promedio de 1.900 granjas avícolas". 
Al realizar la aplicación de los instrumentos de investigación, y luego de procesar los datos, se pudo determinar que el nivel de innovación en la empresa avícola es medio, así lo reconoce el $71 \%$ de los encuestados, mientras que el $25 \%$ del personal considera que la innovación es alta, y, por último, el $4 \%$ de los encuestados manifestó que el nivel de innovación es bajo.

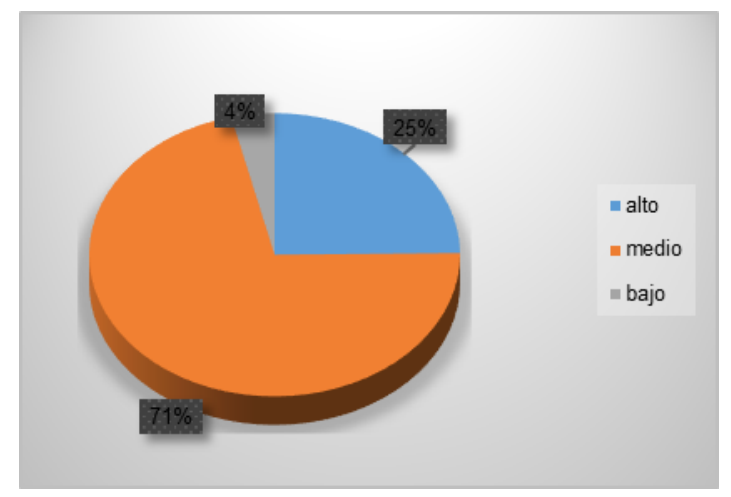

Gráfico 6. Nivel de innovación

Fuente: Guía de Encuesta

Referente a la aplicación de métodos en los procesos operativos y/o sistemas innovadores de producción, se evidenció una debilidad en cuanto a la implementación de éstos, el resultado de la encuesta arrojó que el $59 \%$ del personal manifiesta que el área de producción se aplican métodos o sistemas innovadores de forma parcial, el 32\% respondieron que el área de producción no aplica estos métodos o sistemas innovadores, mientras que el restante $9 \%$ indicaron que si se aplican.

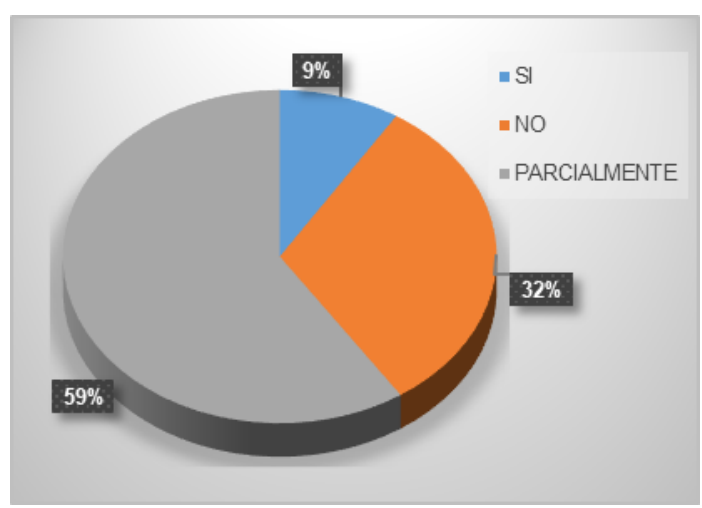

Gráfico 7. Aplicación de métodos y/o sistemas innovadores Fuente: Guía de Encuesta

102

Revista científica Ciencia y Tecnología Vol 20 No 26 págs. 96-105 http://cienciaytecnologia.uteg.edu.ec 
El uso de los indicadores de gestión, constituyen un elemento fundamental para evaluar el que en la empresa avícola no se utilizan los indicadores de gestión, el $67 \%$ del personal encuestado indicó no utilizarlos; mientras que el $25 \%$ respondió utilizarlos parcialmente y el $8 \%$ dijo si utilizar estos indicadores.

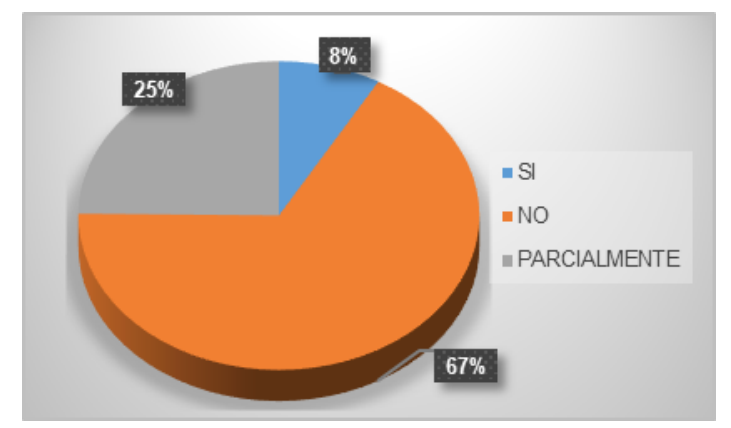

\section{Gráfico 8. Aplicación de métodos y/o sistemas innovadores}

Fuente: Guía de Encuesta

Con el propósito de mejorar la viabilidad económica de la empresa avícola GENETSA para asegurar la perduración de la misma en el mercado, se diseñó un plan estratégico mediante la utilización de una metodología de evaluación de las brechas existentes y de las estrategias que pueden resolver estas brechas para cumplir los objetivos estratégicos de la organización para de esta manera mejorar la rentabilidad de la empresa GENETSA. Considerando el modelo estratégico de Kaplan y Norton, se establecieron los siguientes objetivos:

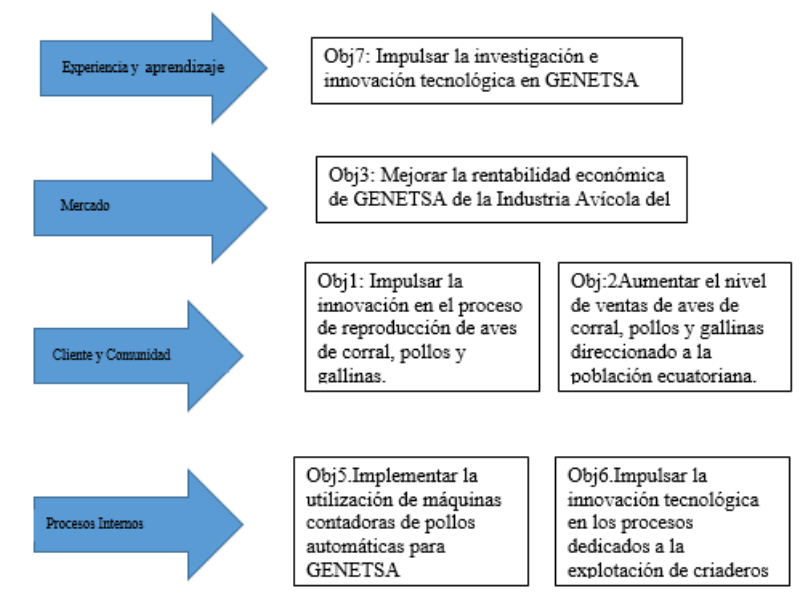

\section{Gráfico 9. Mapa estratégico de objetivos e indicadores \\ Elaborado por: Enma Susana Reyes Domo}




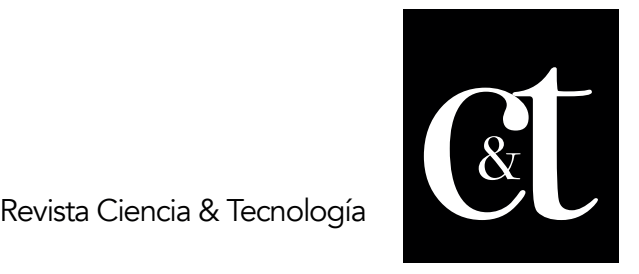

No. 26, 30 de abril de 2020

ISSN impreso: 1390 - 6321

ISSN online: 2661 - 6734

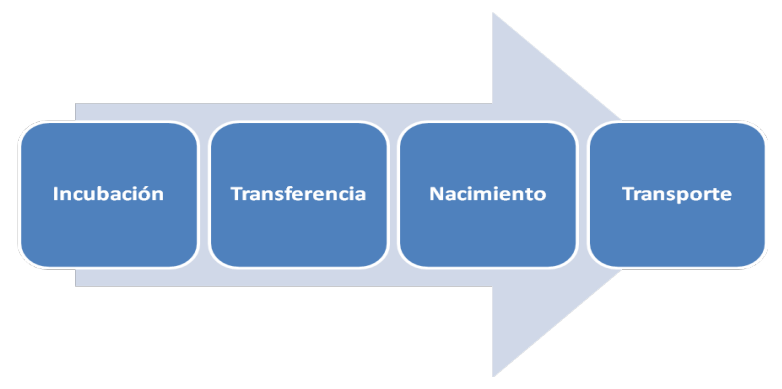

\section{Gráfico 10. Diagrama de procesos de Kaplan y Norton}

Elaborado por: Elaboración propia

\section{Conclusiones}

Las perspectivas reales de crecimiento del mercado avícola en el Ecuador vuelven imperiosa la necesidad de que Genética Nacional continúe con el mismo ritmo de crecimiento sostenido durante los últimos años. Esto avalado por la buena aceptación de sus productos, el respaldo y confianza de sus proveedores, la solidez y transparencia con la que ha manejado sus cuentas, además de la demanda del producto por parte de actuales y nuevos clientes. Hacen que los nuevos proyectos de expansión sean viables desde el punto de vista de la rentabilidad.

La cría intensiva de pollos de engorde cada día es más condicionada, lo que conlleva a los productores ser más eficientes en toda la cadena de crianza, la misma que se debe producir a bajo costo en el producto final. Además, se realizó un diagnostico interno y externo, donde se identificaron aspectos relevantes en cada uno de ellos, llegando a concluir sobre la metodología de trabajo para el mejoramiento organizacional con la implementación de un plan estratégico que contribuirá al crecimiento factible de la empresa, a través de la aceptación de las medidas que se tome en el futuro.

\section{Referencias bibliográficas}

Aillón, M. (2012). Propuesta e implementación de un proyecto comunitario que se dedicara a la crianza, producción y comercialización avícola. Obtenido de http://www.dspace.uce.edu.ec/bitstream/25000/1473/1/T-UCE-0003-272.pdf

Aponte, G. (2015). El proceso de gestión de la innovación Tecnológica sus etapas e Indicadores Relacionados. Revista Venezolana de Análisis de Coyuntura, 5990.

Barbosa, B., \& Dominique, S. (2012). La innovación de los procesos. Estudios y Perspectivas en Turismo, 963-976.

Barzallo, D. (2019). Análisis de la Innovación Tecnológica Avícola Ecuatoriano en el Contexto De Industria 4.0. INVESTIGACIÓN TECNOLÓGICA IST CENTRAL TÉCNICO.

Revista científica Ciencia y Tecnología Vol 20 No 26 págs. 96-105 http://cienciaytecnologia.uteg.edu.ec 


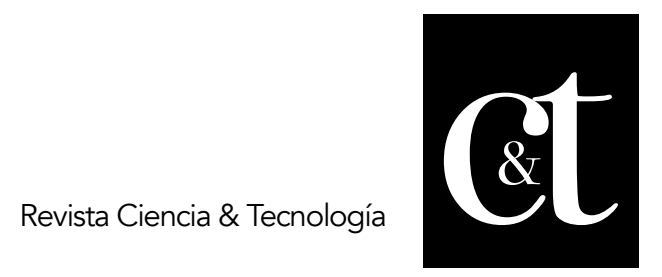

No. 26, 30 de abril de 2020

ISSN impreso: 1390 - 6321

ISSN online: 2661 - 6734

Calle, C., Estrada, M., \& Agudelo, G. (2015). Construcción de un índice de competitividad. Lecturas de Economía , 193-228.

Galeano, L. (2014). Caracterización de sistemas de producción avícola de huevo mediante la implementación de modelos de predicción y clasificación. Obtenido de Universidad de antoquia:

http://bibliotecadigital.udea.edu.co/dspace/bitstream/10495/3096/1/LuisFern ando 2014Caracterizaci\%C3\%B3nSistemasproducci\%C3\%B3n.pdf

Hinojosa. (2006). Cuadernos de Gestión de Tecnología: Innovación de Proceso. Premio Nacional de Tecnología, México, 4.

Jaramillo, M. (2017). Innovación Tecnológica aplicada al sector avicola por sinergia. Obtenido de Avinews: https://avicultura.info/innovacion-tecnologicaaplicada-al-sector-avicola/

Llaque, L. (2009). Innovación en la industria avícola peruana de broilers para mejorar los nivelse de competitividad 1986-2006. Obtenido de Universidad Mayor San Marcos:

http://cybertesis.unmsm.edu.pe/bitstream/handle/cybertesis/3339/Llaque rl. pdf? sequence $=1$ \&isAllowed $=y$

Poultry, (2019). APP para agricultura. Obtenido de http://avicultura.proultry.com/categorias/servicios-varios-para-elsectoravicola/apps-para-aviculturaQuezada

M., Neri , J., \& Pérez, J. (2015). Aprendizaje y talentos de los administradores de granjas avicolas para la Innovación. Revista de Ciencias Ambientales y Recursos Naturales, 75-89.

Revista Productor Agropecuario. (2019). Avicultura y su desafío con las nuevas tecnologías digitales. Obtenido de https://revistaproagro.com/avicultura-y-sudesafio-con-las-nuevas-tecnologias-digitales/

Rio, E. d. (2018). Importancia de la innovación organizativa. Obtenido de http://webs.ucm.es/BUCM/tesis//19972000/S/2/S2033201.pdf

Robayo, P. (2016). La innovación como proceso y su gestión en la organización: una aplicación para el sector gráfico colombiano. ELSEVIER, 125- 140.

Santillán, J. (2014). Modelo de responsabilidad social corporativa en Avesca Avícola Ecuatoriana C.A. y su costo de implementación. Obtenido de Pontificia Universidad Católica del Ecuador: http://repositorio.puce.edu.ec/handle/22000/11703 